\title{
Research Progress on Dialectical Treatment of Cold Coagulation Type Primary Dysmenorrhea of Traditional Chinese and Western Medicine
}

\author{
Huifang Zhu ${ }^{1, a}$, Dongjie Wang ${ }^{1, b}$, Guifeng $\mathrm{Lu}^{1, \mathrm{c}}$, Hang Sun ${ }^{1, \mathrm{~d}}$, Yujing Zhao ${ }^{1, \mathrm{e}}$, \\ Lihua $\mathrm{Niu}^{1, \mathrm{f}}$, \\ ${ }^{1}$ Langfang Health Vocation College, Langfang, Hebei, 065001 \\ ${ }^{\mathrm{a}}$ email, ${ }^{\mathrm{b}}$ email, ${ }^{\mathrm{c}}$ email, ${ }^{\mathrm{d}}$ email, ${ }^{\mathrm{e}}$ email, ${ }^{\mathrm{f}}$ email
}

Keywords: Cold Coagulation Type; Primary Dysmenorrhea; Chinese and Western Medicine

\begin{abstract}
At present, there are few studies on the treatment of primary dysmenorrhea of cold coagulation type primary dysmenorrhea with traditional Chinese and western medicine at home and abroad. The study of blood deficiency and cold coagulation type of primary dysmenorrhea are alpine stagnation with qi and blood deficiency type, it should be nourishing blood stasis and relieve pain, warm by cold and warm uterus. Angelica sinensis plus Evodia ginger soup with nourishing meridians, cold and back to the role of cold, for the blood deficiency caused by cold coagulation of primary dysmenorrhea have a certain therapeutic effect. Warming soup can nourishing blood stasis, temperature and cold, attending congestion block and deficiency and other evidence, also rule the woman uterus cold, the prescription for blood deficiency caused by the cold type of primary dysmenorrhea also have some treatment effect
\end{abstract}

\section{Introduction}

Primary dysmenorrhea can also be called functional dysmenorrhea, mainly refers to the female menstrual period focused on the lower abdomen spastic pain. Primary dysmenorrhea symptoms include dizziness, nausea, vomiting, low back pain, fatigue and other symptoms, is a more common disease of young women, not accompanied by significant pelvic inflammatory disease, endometriosis and tumors such as pelvic organic disease. The main causes of primary dysmenorrhea are mostly due to the following: uterine hypoplasia, membranous dysmenorrhea, uterine flexion, uterine hypoplasia, poor physical and physical factors, mental factors and allergic reactions.

\section{The Blood Deficiency Cold Coagulation Type of Primary Dysmenorrhea of Integrative Medicine}

Dysmenorrhea is more common in obstetrics and gynecology, the pathogenesis of the disease complex, more incentives, severe fainting occurs, to the patient's life and work caused great difficulties. Primary dysmenorrhea in the clinical gynecology is also more common, the pathogenesis of the disease complex, more incentives, severe cases of syncope will occur to the patient's life and work caused great distress. Clinical treatment of primary dysmenorrhea is often difficult to achieve the desired effect of Western medicine, ibuprofen commonly used in oral treatment although it can reduce the production of prostaglandins, analgesic effect is better, but gastrointestinal reactions, skin rash, headache, etc. Adverse reactions more, not long-term use, and active peptic ulcer patients and patients allergic to ibuprofen should not be taken. For blood deficiency type of primary dysmenorrhea according to syndrome differentiation using traditional Chinese medicine prescription treatment can solve the problem of Western medicine treatment to achieve the effect of tackling the problem. But the slow effect of traditional Chinese medicine, it is difficult to quickly help patients to relieve pain and pain, so there is no active peptic ulcer patients and patients with no allergic reactions to ibuprofen cold primary pain patients with traditional Chinese and Western medicine treatment Better results, more clinical value. 


\section{The Status Quo and Development Trends At Home and Abroad}

Epidemiological survey showed that the incidence of dysmenorrhea in women in China about $33.01 \%$, of which the incidence of primary dysmenorrhea accounted for 55.2\% [1]. Up to now, modern medicine has been recognized prostaglandin and interleukin increased primary dysmenorrhea is the main pathogenesis [2]. Akdemir et al [3] pointed out that primary dysmenorrhea is also associated with vascular endothelial dysfunction. Lejie pointed out that the primary dysmenorrhea before or after menstruation or menstrual period in the absence of organic genital lesions occurred under the abdominal pain and discomfort; secondary dysmenorrhea as pelvic inflammatory disease, endometriosis and other pelvic devices Qualitative lesions caused by dysmenorrhea. At present, the clinical treatment of primary dysmenorrhea this disease has not been an ideal treatment, the traditional method of treatment mainly to Western-based [5]. The clinical application of non-steroidal anti-inflammatory drugs, although these drugs can effectively inhibit the synthesis of PG, reduce the tension and contraction of the uterus in patients with primary dysmenorrhea, effective in reducing the symptoms of dysmenorrhea, but these drugs on patients There are certain side effects, and long-term clinical effects of treatment is not ideal, most patients will have a relapse after stopping the situation, to patients with physical and mental pain caused great [6]. Primary dysmenorrhea is still not entirely clear reason for the onset, Western medicine that the incidence of primary dysmenorrhea is due to menstrual endometrial release of prostaglandin caused by excessive uterine contraction caused by strong [7]. Chinese medicine that the primary dysmenorrhea are "by the line of abdominal pain," the scope of the pathogenesis is due to cold stagnation or Chong Ren Bu Tiao and other factors caused by poor local blood, Chinese medicine clinical primary dysmenorrhea is divided into alpine Stagnation, stagnation of qi and blood, qi and blood deficiency, liver and damp heat, liver and kidney losses five card type [8]. "Nei Jing" cloud "hot blood is the line, when the cold is astringent and not", people injured in the cold or because of Tan Liang or exogenous cold blood and blood Yin Yin, loss and Chong Ren of the pulse, not General pain. "The source of Hou theory," proposed that the reasons for women's abdominal pain is cold in the Chong Ren Ren pulse, saying: "Women on the water to abdominal pain, blood gas from the injured workers, so physically weak, by air-cooled gas, In the treatment of dysmenorrhea on the dialectical treatment of that: "by the line of abdominal pain, the card has the actual situation." Real or because the cold stagnation of the cold, Or because of blood stagnation, or due to qi stagnation, or due to thermal stagnation; deficiency due to blood deficiency, due to qi deficiency. "" Fu Qing master women "said" husband cold evil is evil, Under the coke, "the primary dysmenorrhea more real less virtual, and often for qi stagnation, blood stasis, cold condensate phase with the disease, each other cause and effect. Gu Shimin points that [9], the combination of Chinese and Western medicine treatment of dysmenorrhea can solve the problem, while making up for the slow onset of Chinese medicine. Wang Hui [10] takes 60 cases of primary dysmenorrhea patients with integrated traditional Chinese and Western medicine treatment and care to obtain a satisfactory effect. Xiaoxiao Tao [11] on 90 cases of alpine stagnation syndrome, damp heat silted stagnation syndrome, qi deficiency syndrome, liver and kidney deficiency dysmenorrhea primary dysmenorrhea according to TCM treatment, the treatment results show that traditional Chinese and Western medicine treatment of primary dysmenorrhea cure rate, Consolidation of lasting effect, the overall clinical efficiency is higher. Yang Haiping [12] on 78 cases of primary dysmenorrhea in patients with traditional Chinese and Western medicine combined treatment to obtain a certain effect. Wu Jinping et al [13] on 51 cases of qi stagnation and blood deficiency in patients with primary dysmenorrhea by Xuefu Zhu soup with ibuprofen sustained-release capsule treatment, and achieved good results. So far, few studies at home and abroad for the cold blood coagulation dysmenorrhea type of primary and secondary treatment of dysmenorrhea related research.

The study of blood deficiency and cold coagulation type of primary dysmenorrhea are alpine stagnation with qi and blood deficiency type, should be nourishing blood stasis and relieve pain, warm by cold, Fuyang Warm Uterus based. In this study, the prescription of Danggui Sini Decoction, Wuzhuyu Ginger Soup and "Daquan Prescription of Wenshenwang" were used in the 
treatment of "Shanghan Miscellaneous Diseases": "Hand, Foot and Jue , Veins stricter who, Angelica Sini Decoction of the main; if its people who have a long cold, Angelica Sini plus Evodia ginger soup main. "" Treatise on Fang "in the cloud:" There is a long cold, A Yang is not enough for the Kai Tai of the, and the meridian of the virtual, is due to even so. Therefore to Evodia, Asarum and reverse the Runzao, through the grass for the lead, complex to Guizhi Quan Tang and Jun to Angelica, Can be seen, Angelica Sini plus Evodia ginger soup with nourishing meridians, cold and back to the role of cold, for the primary cause of blood deficiency cold congeals Of dysmenorrhea have a certain therapeutic effect. Warming soup can nourishing blood stasis, temperature and cold, attending congestion block and Chong Ren Deficiency and other evidence, also rule the woman Gongleng, the prescription for blood deficiency caused by the cold type of primary dysmenorrhea also have some treatment effect. Chuanxiong played a blood Tiaojing, Huoxue analgesic effect on the uterus are excited and inhibit the two effects; Cyperus rotus can help patients Shugan Qi, menstruation pain, effective To ease the tension of the uterine muscle, play the role of analgesic, anti-inflammatory effect; Guizhi, ginger temperature and pain, peony nourishing and relieving pain, Evodia warm liver pain, fennel, cinnamon into the coke to the cold temperature by pain, Wulingzhi, Puhuang can Huayu pain; Salvia blood Tiaojing, Tong grass-benefit, Rehmannia benefits liver and kidney, Atractylodes spleen Qi, licorice to reconcile the various drugs, various drugs can be nourishing tonic, warm through Yang, blood circulation blood stasis, blood conditioning, coordination Chong Chong, to alleviate the purpose of dysmenorrhea.

\section{References}

[1] Qin Mingfang, Long Xiuhong, Li Weihong. Effect of combination of traditional Chinese and western medicine for primary dysmenorrheal combined with evidence-based nursing intervention [J]. Guangxi Medical, 2015, 7 (37): 1025-1026.

[2]Harel Z. Dysmenorrhea in adolescents and young adults: from pathophysiology to pharmacological treatments and management strategies [J]. Expert Opinion Pharmacother, 2008, 9 (15): 2661-2672.

[3] Ardemir N, Cinemre H, Bilir C, et al. Increased serum asymmetric dimethylarginine in primary dysmenorrhea [J]. Gynecal Ob-stet Invest, 2010, 69 (3): 153-156.

[4] Le Jie. Obstetrics and Gynecology [M]. 7th ed., Beijing: People's Medical Publishing House, 2008,318.

[5] Tanwang Jianjun, Huang Yanqing. Chinese Journal of Metallurgical Industry Medicine, 2015,3 (32): 268-269.

[6] Tan Peihui. Clinical study on 130 cases of dysmenorrhea treated with traditional Chinese and western medicine [J]. China Modern Medicine Application, 2014,13 (8): 141-143.

[7] Zhang Xiaoling, Zhang Shijun, Xu Chengkang. Decoction combined with moxibustion treatment of primary dysmenorrhea 55 cases [J]. Chinese Journal of Experimental Traditional Medical Formulas, 2014,20 (16): 192-195.

[8] An Ping. Etiology of dysmenorrhea compared with traditional Chinese and Western medicine treatment [J]. Inner Mongolia Traditional Chinese Medicine, 2013,6 (10): 26-28.

[9] Gu Shimin. Observation on the curative effect of integrated traditional Chinese and western medicine on dysmenorrhea [J]. Chinese Journal of Integrative Medicine on Cardiovascular Diseases, 2015,3 (13): 26-28.

[10]. Wang Hui. Observation on 40 cases of primary dysmenorrhea treated by combination of traditional Chinese and western medicine and nursing [J]. Journal of Yangtze University (Natural Science Edition), 2015, 12 (12): 82-83.

[11]. Xiao Xiaotao. Observation on the curative effect of integrated traditional Chinese and western medicine on primary dysmenorrhea [J]. New Chinese Medicine, 2013, 9 (45): 81-83. 
[12]. Yang Haiping. Observation on the curative effect of integrated traditional Chinese and western medicine on primary dysmenorrhea [J]. Modern medicine and health, 2015,17 (31): 2653-2655.

[13]. Wu Jinping, Kang Zhiyuan. Chinese and Western medicine treatment of primary dysmenorrhea clinical study [J]. Chinese Journal of Traditional Chinese Medicine, 2014, 6 (29): 896-899. 